Research Article

\title{
Multiobjective Multistate System Preventive Maintenance Model with Human Reliability
}

\author{
Chao-Hui Huang $\mathbb{D},{ }^{1}$ Chun-Ho Wang $\mathbb{D},{ }^{2}$ and Guan-Liang Chen $\mathbb{D}^{2}$ \\ ${ }^{1}$ Department of Applied Science, R.O.C. Naval Academy, Kaohsiung 813205, Taiwan \\ ${ }^{2}$ Chung Cheng Institute of Technology, National Defense University, Taoyuan 335009, Taiwan \\ Correspondence should be addressed to Chao-Hui Huang; a0970437433@gmail.com
}

Received 25 December 2020; Revised 11 April 2021; Accepted 30 June 2021; Published 14 July 2021

Academic Editor: Maj D. Mirmirani

Copyright (c) 2021 Chao-Hui Huang et al. This is an open access article distributed under the Creative Commons Attribution License, which permits unrestricted use, distribution, and reproduction in any medium, provided the original work is properly cited.

\begin{abstract}
Modern equipment is designed to operate under deteriorating performance conditions resulting from internal ageing and/or external environmental impacts influencing downstream maintenance. This study focuses on the development of a multistate system (MSS) that considers a human reliability factor associated with maintenance personnel-a condition-based multiobjective MSS preventive maintenance model (MSSPMM). The study assumes that no more than one maintenance activity is performed to achieve the most appropriate preventive maintenance (PM) strategy and easy implementation and to reduce maintenance error due to human reliability. The MSS performance based on mean system unavailability and total maintenance cost is evaluated using a stochastic model approach, and then, the MSSPMM is used for optimisation. A customised version of the nondominated sorting genetic algorithm III is employed to ensure efficient solution of the PM model with human reliability-which is considered a constrained multiobjective combinatorial optimisation problem. The optimised solutions are determined from the nondominated Pareto frontier comprising the diversified PM alternatives. A helicopter power transmission system is used as an example to illustrate the efficacy and applicability of the proposed approach through sensitivity analyses with relevant parameters.
\end{abstract}

\section{Introduction}

Human reliability plays a critical role in the design, manufacture, and operation of structures, machines, or devices in fields such as aviation safety [1], nuclear power plant safety [2], and medical services [3, 4]. Heo and Park [2] used nuclear power plant safety as an example to demonstrate that errors made by maintenance personnel significantly increase overall system costs and result in accidents. Guo et al. [1] proposed a quantitative model that modifies the cognitive reliability and error analysis method pioneered by Hollnagel for the nuclear power industry [5] to predict the probability of human error in flight. They showed that missed actions by the pilot are the most critical cognitive function influencing human reliability. Kalaiarasi et al. [3] incorporated the human error rate of technicians performing a task to establish the failure probability of a clinical laboratory over time. Zaitseva et al. [4] considered the effect of human factors on complications in the familiarisation and exploitation of a new device for healthcare. They proposed a structure function construction method for analysing human reliability that maps all possible component states onto system performance levels. Asadzadeh and Azadeh [6] showed that maintenance personnel reliability, including diagnostic errors and prognostic errors, adversely affects the overall multistate system (MSS) cost in a condition-based preventive maintenance (PM) strategy.

A helicopter power transmission system relies on regular maintenance to ensure safe and reliable operation under conditions such as vibration, pollution, and wear, which can gradually degrade its performance. An appropriate maintenance strategy is essential as over- or undermaintenance can result in aviation risks and increase maintenance costs. Personnel management is also critical for minimising maintenance faults [7]. In this regard, Chen and Yu [8] adopted a human factor intervention matrix considering feasibility, 


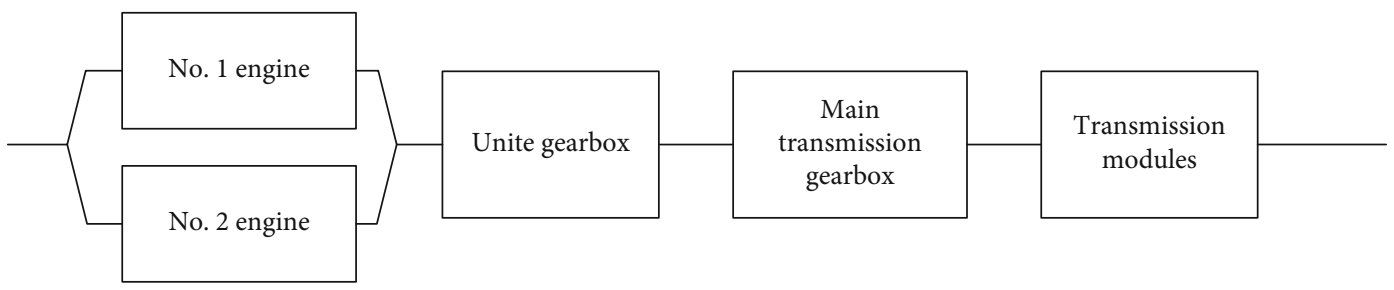

FIGURE 1: Configuration of helicopter power transmission system.

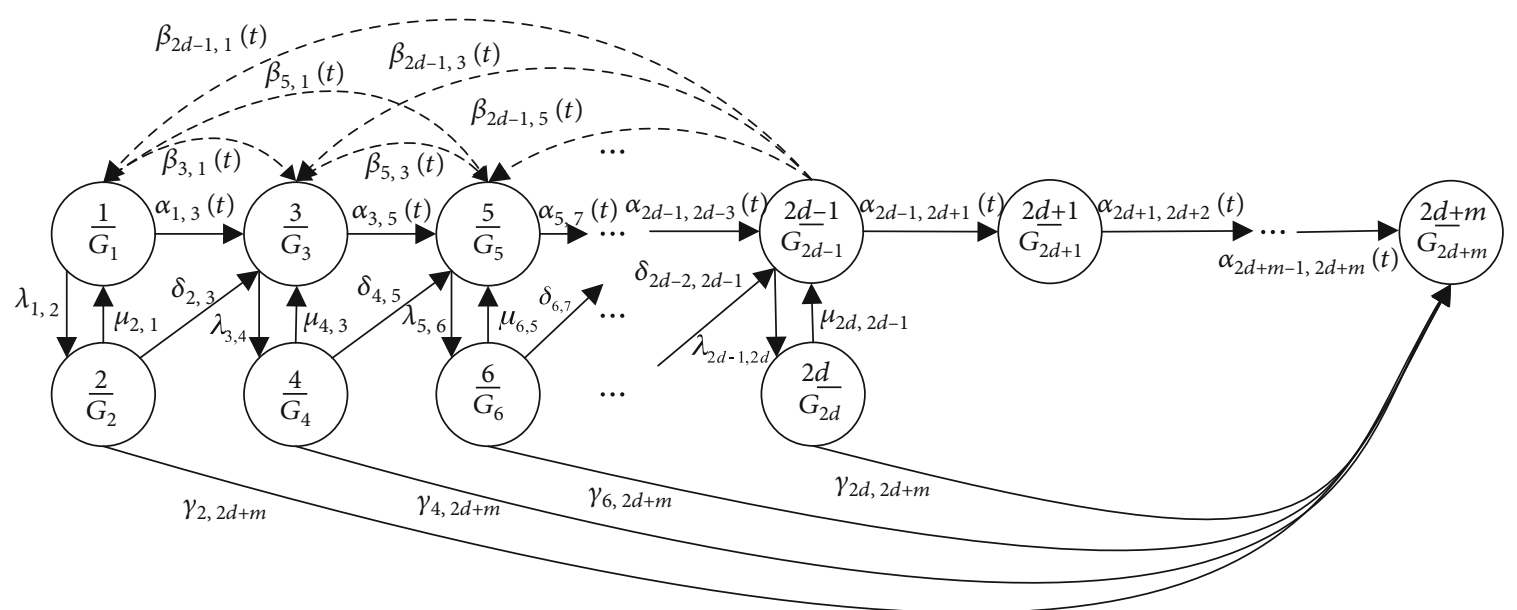

Figure 2: Transition diagram of repairable multistate assembly.

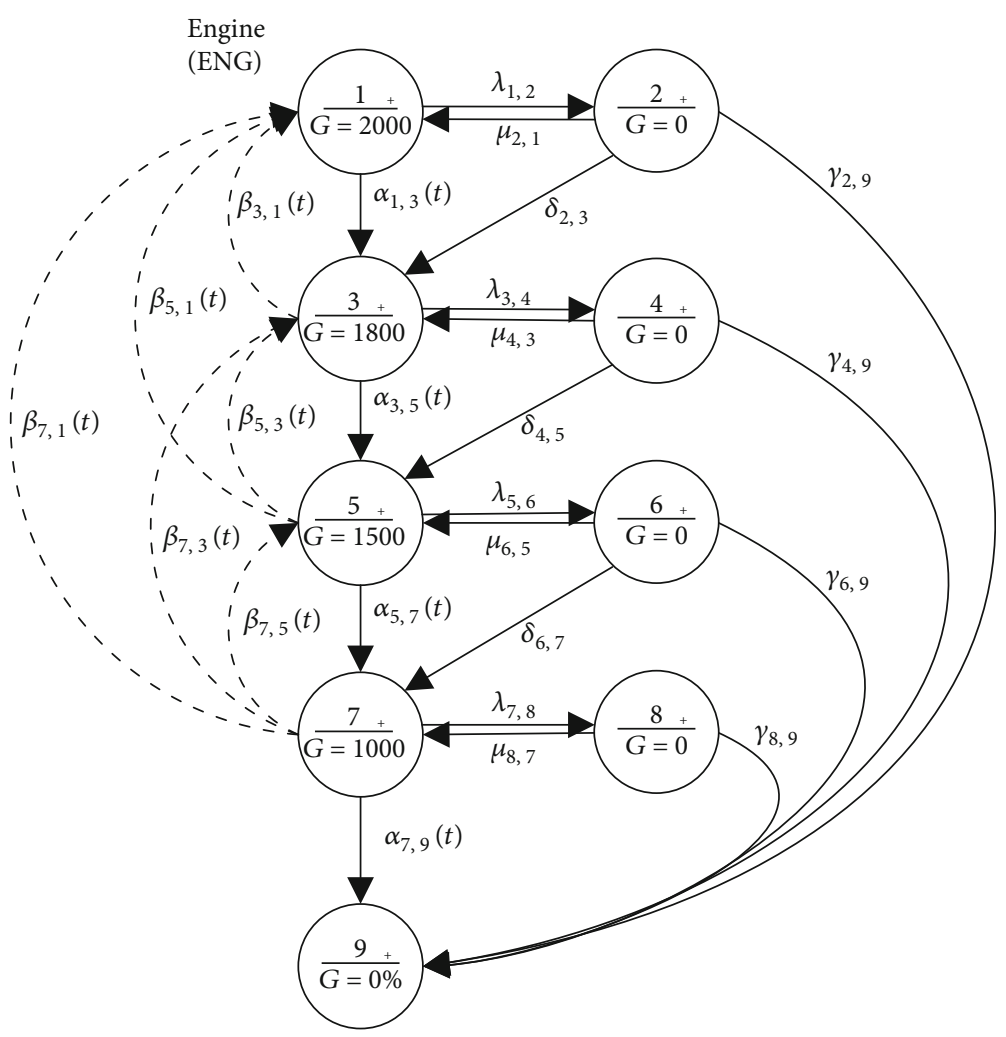

FIgURE 3: State-transition diagram of engine. 


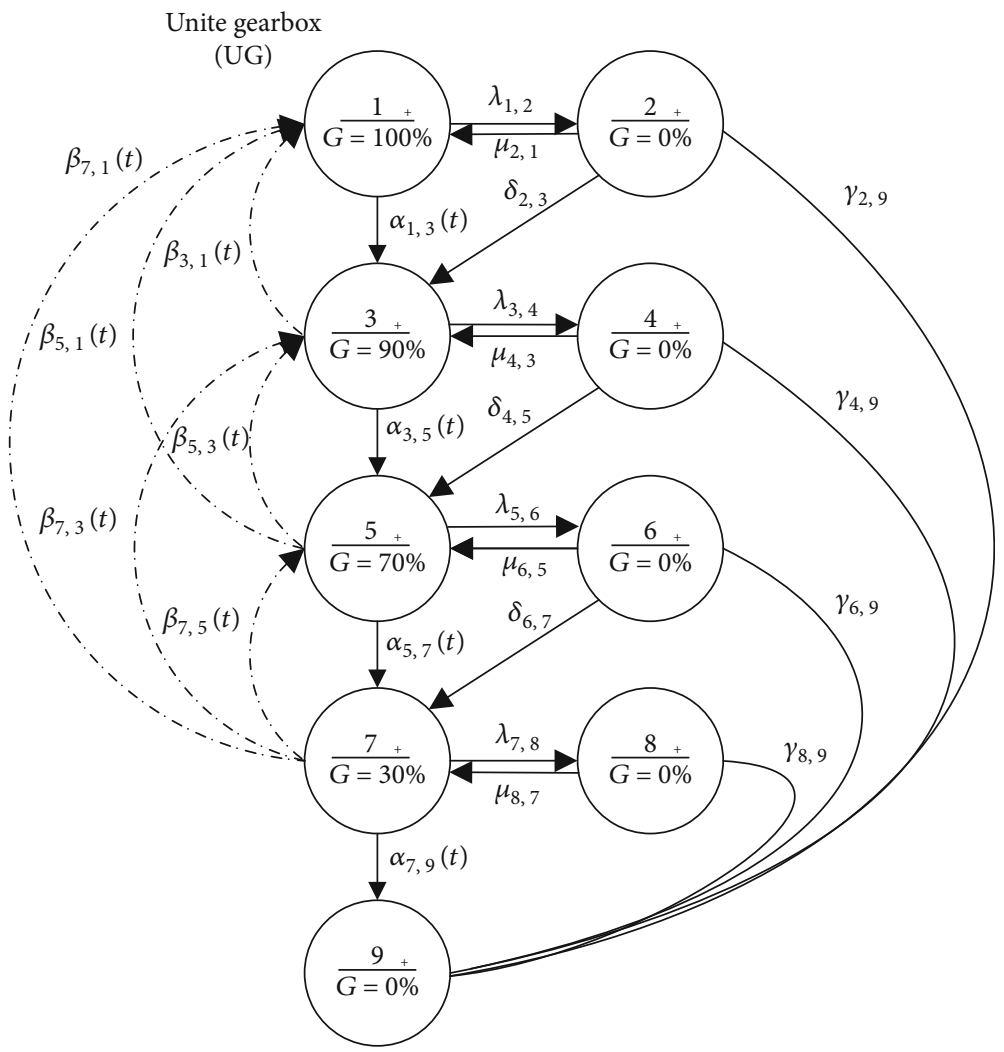

FigURE 4: State-transition diagram of unite gearbox.

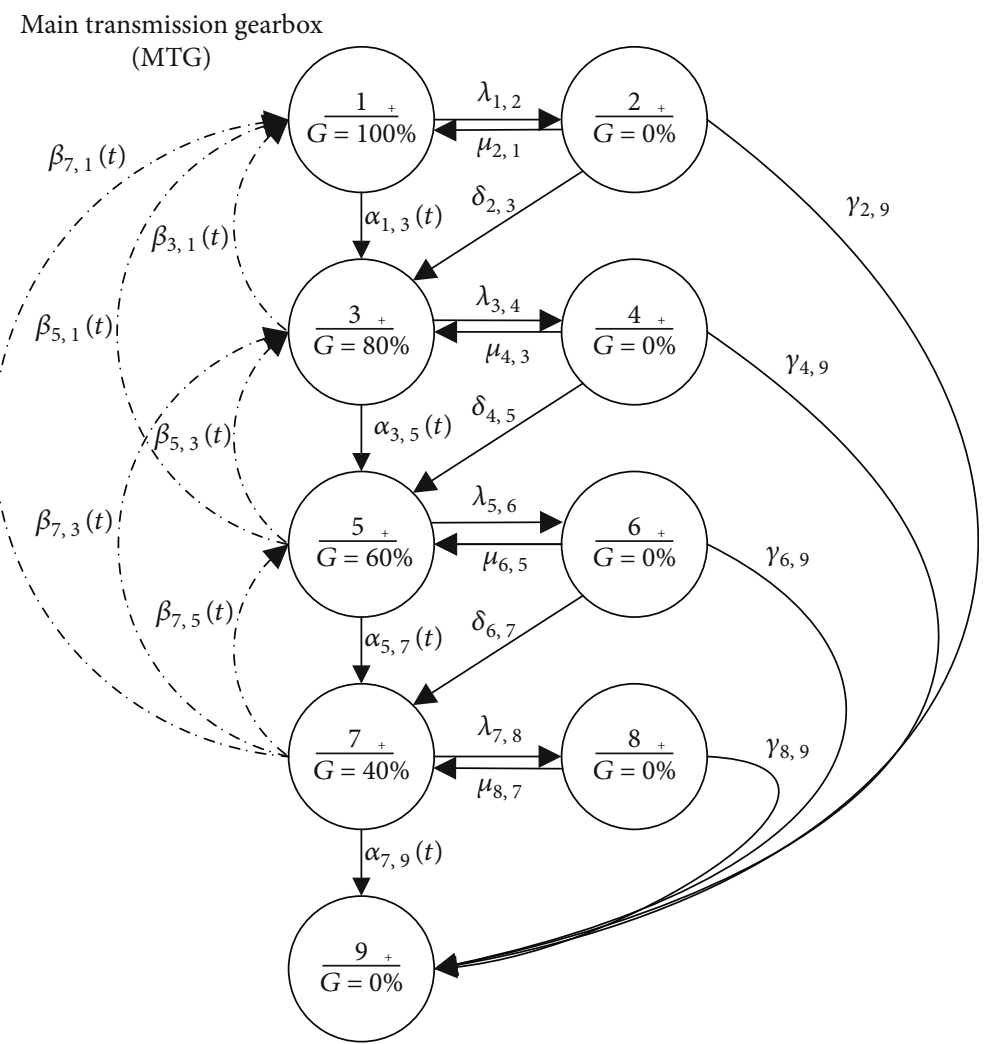

FiguRE 5: State-transition diagram of main transmission gearbox. 
Other transmission modules (OTM)

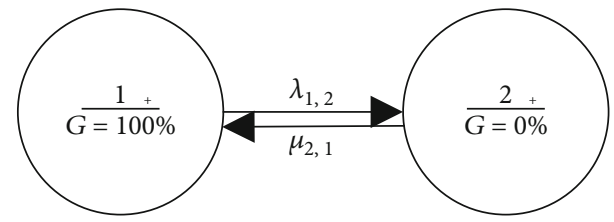

FIGURE 6: State-transition diagram of other transmission modules.

acceptability, effectiveness, and sustainability to develop criteria for an intervention strategy model that can alleviate unsafe maintenance behaviours.

Many studies have employed stochastic models to quantify the dynamic behaviour of an MSS [9-12]. This study employs ageing multistate performance characteristics to establish a multistate system preventive maintenance model (MSSPMM) that considers human reliability, which is a topic that has received increasing research attention [13-15]. MSSPMM constrains maintenance activity in the degraded states of the components to only one, making the maintenance strategy simple and viable to avoid unsafe maintenance behaviours. The goal of this study is to improve the efficacy of the maintenance strategy of a helicopter power transmission system.

PM strategies can be categorised into time- and condition-based maintenance strategies. Condition-based maintenance strategies comprise diagnosis and prognosis, and they determine the most appropriate maintenance activities by monitoring the status of the equipment. Previously reported studies indicated that condition-based maintenance strategies outperform their traditional time-based counterparts in terms of equipment quality, reduction in life-cycle costs, and major failure avoidance. Zhao et al. [16] proposed a condition-based PM strategy to predict the remaining lifetime of aircraft engines. Moghaddam [17] focused on a CNC machine in a tandem system for constructing a multiobjective PM and replacement model from a system perspective based on a binary reliability theory with operating costs and system reliability as biobjectives. Salmasnia et al. [18] established a multiobjective PM model with PM conducted only in the final acceptable state. Koutras et al. [19] proposed a condition-based MSSPMM with system availability and operating cost as biobjectives and decision variables covering the inspection cycle, in addition to maintenance activities including imperfect maintenance, minimum maintenance, and major maintenance, as well as maintenance upon failures. A weighted approach that weighs multiple objectives in a single metric $[18,19]$ was employed to solve the proposed biobjective model while being confined mathematically to a single nondominated solution on the efficiency front [20].

The nondominated sorting genetic algorithm III (NSGAIII) [21-23] has been successfully adopted to solve various multiobjective optimisation problems, and variants have been developed for specific problems [24-28]. Bhesdadiya et al. [21] applied NSGA-III to lower the emission value and fuel cost of fossil fuel power plants while providing a sustainable and reliable power supply. Guo et al. [22] proposed a

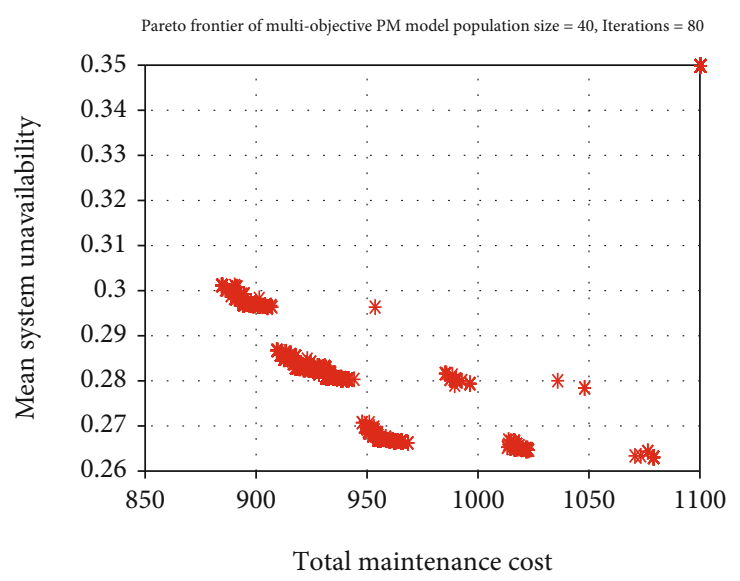

Figure 7: Approaching Pareto frontier.

risk-based multiobjective framework for a fault current limiter (FCL) that employs a data-driven proportional hazard model to consider equipment ageing, weather statistics, and surrounding conditions for evaluating the nodal shortcircuit fault probability. NSGA-III was used to configure the FCL in a more economical manner. Yang and Zhang [23] applied NSGA-III to maximise reliability, minimise cost, and allocate transmission lines efficiently in multistate networks. Li et al. [24] applied NSGA-III to solve a triobjective military optimisation problem for minimising expected damage and operation costs and maximising combat value for fighter jets. Ni et al. [25] applied NSGA-III to optimise the complex coupling relationship between flood control, power generation, and ecological maintenance of a reservoir. $\mathrm{Bi}$ and Wang [26] proposed an improved NSGA-III with enhanced convergence. Liu et al. [27] improved NSGA-III using a genetic $K$-means (GKM) clustering algorithm to improve convergence. Zheng et al. [28] introduced the acceleration of the differential evolution mechanism for improving NSGA-III to accelerate convergence and diversity exploration. Chao [29] showed that the random search algorithm outperforms the gradient-based counterparts including distorted and stochastic distorted greedy algorithms and resolves monotonic approximation with size constraints to maximise the submodular submodulus function. Different algorithms have been developed to solve multiobjective problems. Further, these algorithms address decision variables involved in NP-hard combinatorial problems such as wireless communication networks, data exploration, resource allocation, and traditional travelling salesman problems. When addressing these combinatorial multiobjective optimisation problems, finding a solution set among several conflicting objectives remains a challenge. Zahra et al. [30] verified the efficiency of NSGA-II to solve complex combinatorial optimisation problems. Behmanesh et al. [31] evaluated18 types of evolutionary multiobjective algorithms and confirmed that NSGA-III is effective for optimising NPhard combinatorial optimisation problems.

The proposed biobjective MSSPMM with human reliability for the helicopter power transmission system is a NP-hard combinatorial optimisation problem in which 


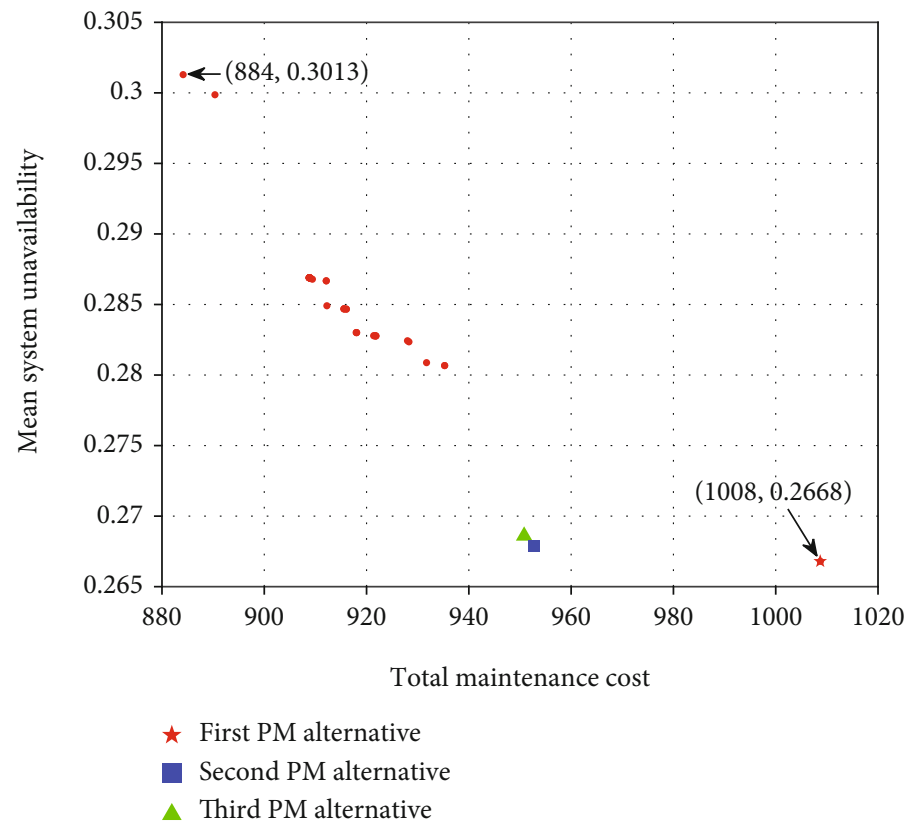

FIGURE 8: Pareto frontier solution set.

TABLE 1: Three PM alternatives with mean system unavailability and total maintenance cost.

\begin{tabular}{lcc}
\hline & $\overline{\mathrm{UA}}$ & $\mathrm{Cs}$ \\
\hline First PM alternative & 0.2668 & 1008 \\
Second PM alternative & 0.2679 & 952 \\
Third PM alternative & 0.2686 & 950 \\
\hline
\end{tabular}

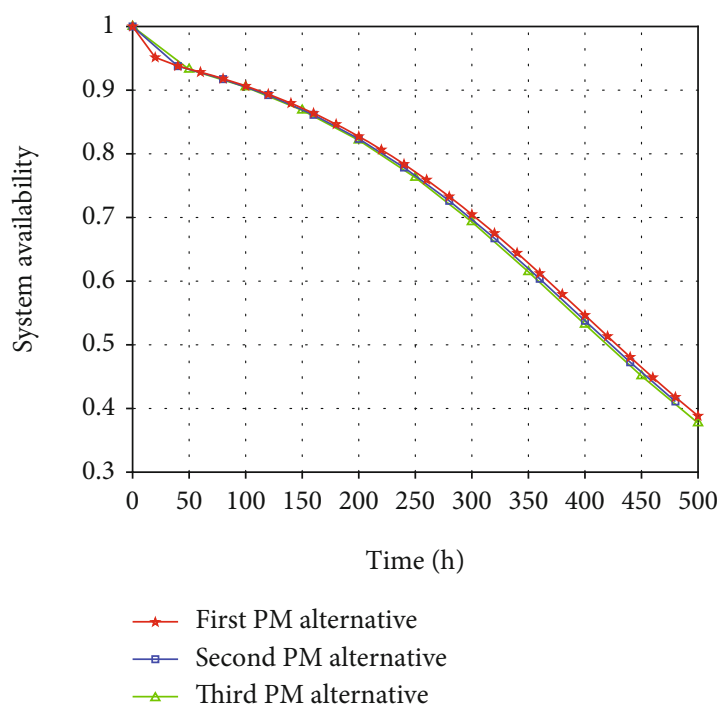

FIGURE 9: System availability trajectory with time.

the total maintenance cost and mean system unavailability are subject to at most one type of maintenance activity. It overcomes the limitations of past studies by not considering human error beyond maintenance activities confined only to the last MSS acceptable state, which simplifies the calculation and optimisation processes [18]. Further, it overcomes the complexity of the MSS performance calculation, which increases exponentially with the addition of components and degradation states [32]. This is achieved by mapping the probability mass functions (PMFs) of all components derived in advance into the system PMF using a universal generation function (UGF) technique that involves simple algebraic calculations [12]. The Pareto frontier solutions obtained from NSGA-III provide various PM alternatives. It is expected that engineers will be able to gain further insight into the resulting solutions and select the most effective alternative based on their preference, limitation of maintenance resources, or system performance.

In summary, human reliability has a profound adverse effect on apparatus performance; however, related research on multiobjective MSSPMMs considering human reliability and ageing effects simultaneously is limited. Considering these negative effects on MSS can reflect actual maintenance scenarios more appropriately and yield more precise MSS reliability performance. Further, it can significantly reduce the maintenance-induced costs, particularly in the aviation and nuclear power industries.

\section{Model Description}

\subsection{Assumptions}

(1) Real-time monitoring of component performance is available

(2) A PM action can be conducted to restore components in acceptable states to a previous better state

(3) A component may feature multistate degradation with distinct performance levels. 


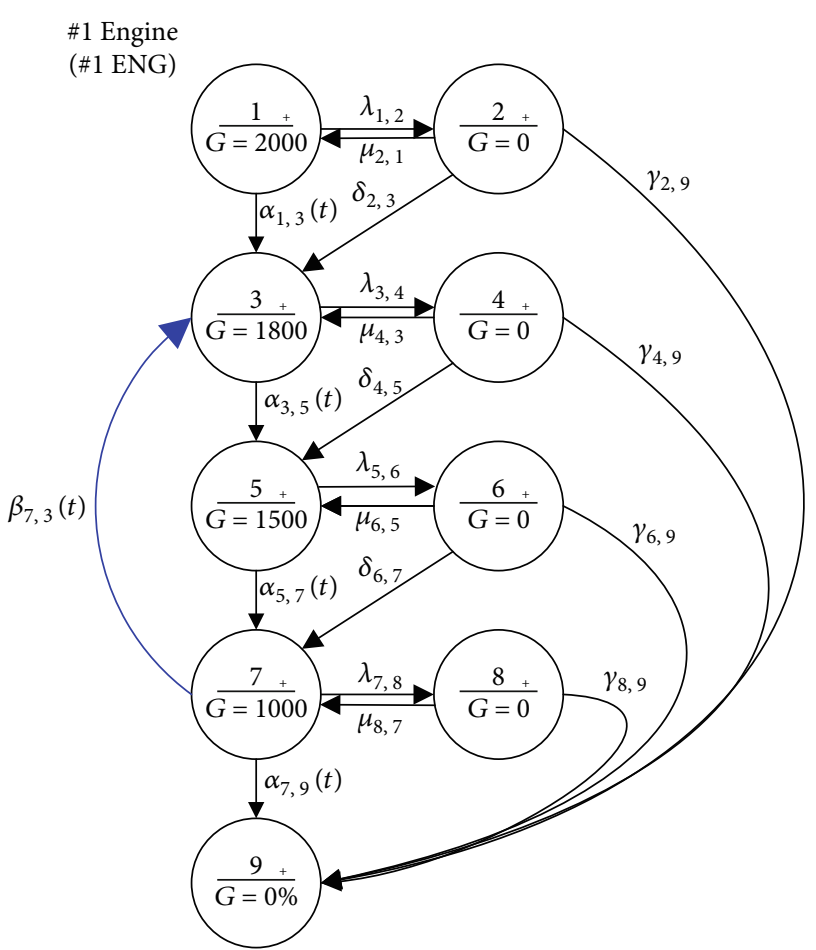

Figure 10: State-transition diagram of No. 1 engine.

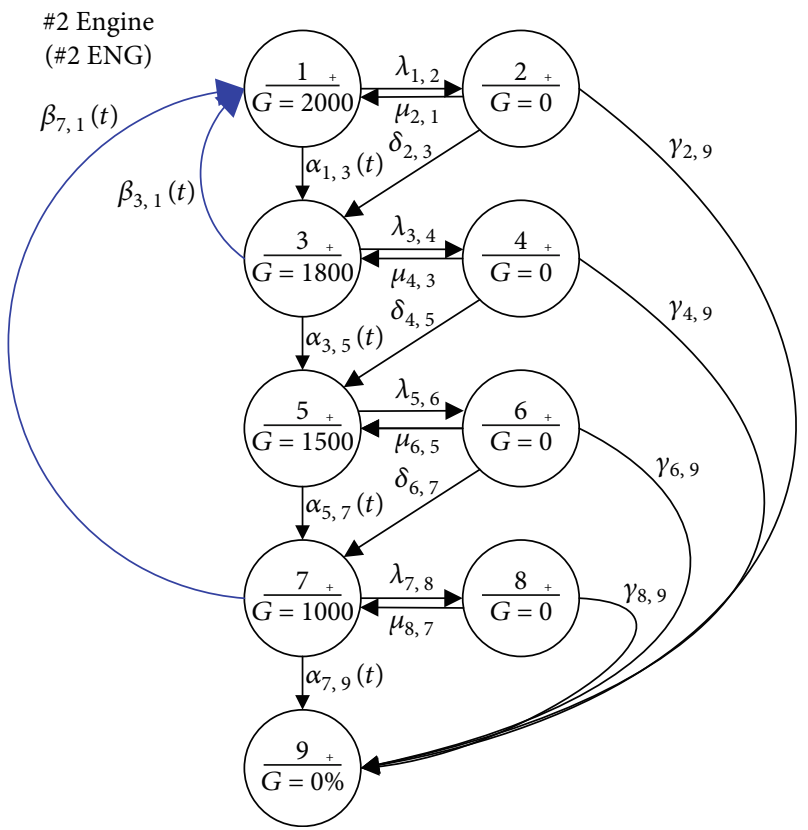

FIGURE 11: State-transition diagram of No. 2 engine.

(4) Minimum repair is conducted when components fail from any operational state

(5) Two types of human reliability are considered in terms of minor and critical human errors

(6) Failure rates and minimum repair rates are distributed exponentially, whereas degradation rates, PM rates, and human error rates feature the ageing property

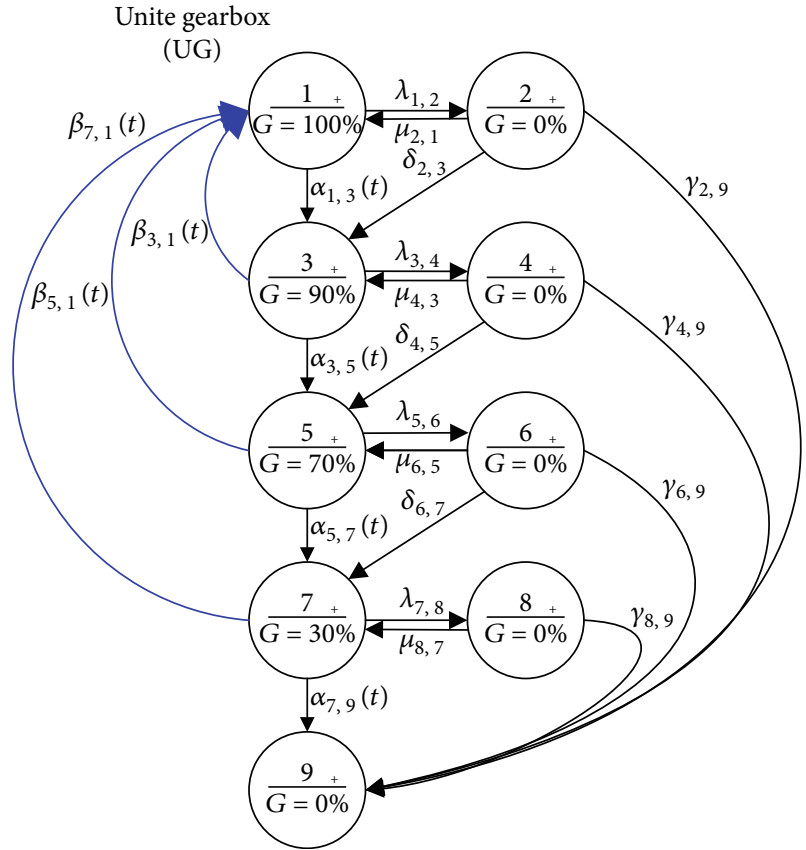

Figure 12: State-transition diagram of the unite gearbox.

(7) The status of each component is independent of that of other components

2.2. Evaluation of MSS Dynamic Performance. Figure 1 shows the configuration of the helicopter power transmission system used as a case study to verify the proposed approach. It comprises two engines (No. 1 ENG and No. 2 ENG) connected in parallel; they are connected in series with the unite gearbox (UG), main transmission gearbox (MTG), and other transmission modules (OTM) to transmit power to other helicopter subsystems. From a perfect state, the assembly in the system degrades to worse states over time, which degrades MSS performance; the system can be restored to previous better states through appropriate PM activities. Further, the assemblies may suffer a random and sudden Poisson failure before minimum repair is conducted, during which human error may occur, which can push the system into the next worse state or even lead to complete failure; the same scenario occurs in all operational states. An assembly that cannot meet the minimum demand is deemed a failure. The PM activities that involve several types of candidates classified into minor maintenance to major maintenance are conducted optionally on operational states to restore the assembly back to previous better states, which helps maintain and improves MSS performance. Figure 2 shows a general state-transition diagram of the assembly using the following notations.

State (1): Perfect functioning,

State $(2 l-1)$ : Degraded states, $l=2, \cdots, d$,

State $(2 l)$ : Failed from operational states, $l=1, \cdots, d$,

State $(2 d+k)$ : Failed after degradation, $k=1, \cdots, m$,

$\lambda_{i j}$ : Failure rate from state $i$ to state $j$, where $i=2 l-1$ and $j=2 l, l=1, \cdots, d$, 


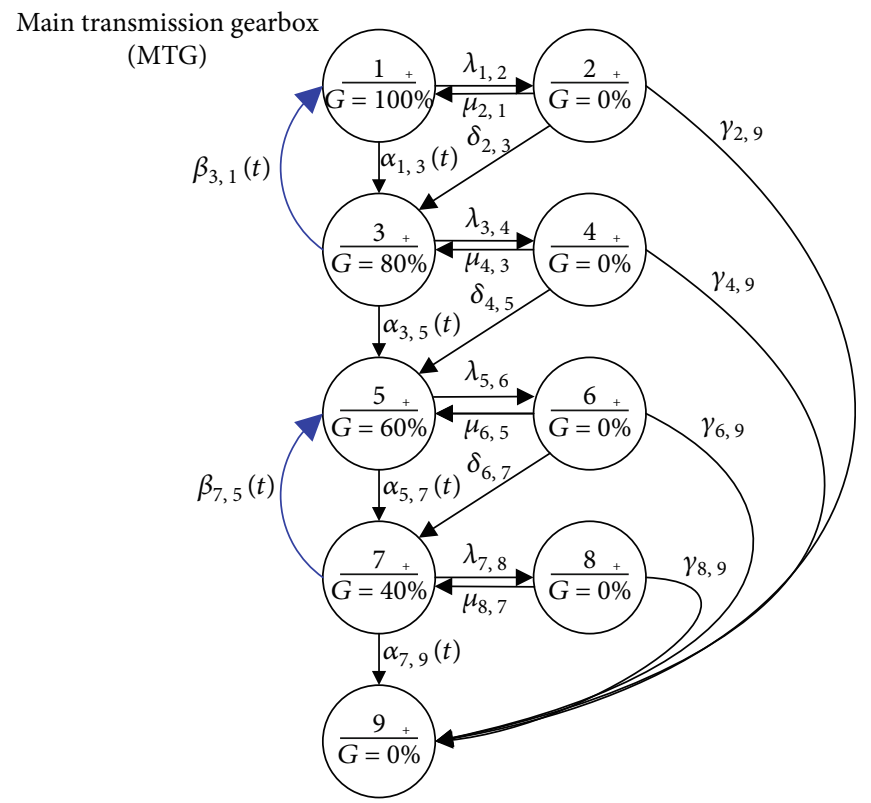

FIgURE 13: State-transition diagram of the main transmission gearbox.

TABle 2: Transition rates of assemblies with exponential distribution.

\begin{tabular}{|c|c|c|c|c|c|}
\hline & & ENG & UG & MTG & OTM \\
\hline \multirow{4}{*}{ Failure rates } & $\lambda_{1,2}$ & 0.0032 & 0.0060 & 0.005625 & 0.005 \\
\hline & $\lambda_{3,4}$ & 0.0040 & 0.0096 & 0.00090 & - \\
\hline & $\lambda_{5,6}$ & 0.0048 & 0.0112 & 0.0105 & - \\
\hline & $\lambda_{7,8}$ & 0.0060 & 0.0144 & 0.0135 & - \\
\hline \multirow{4}{*}{ Minimum repair rates } & $\mu_{2,1}$ & 0.0075 & 0.084 & 0.063 & 0.01 \\
\hline & $\mu_{4,3}$ & 0.0060 & 0.078 & 0.0585 & - \\
\hline & $\mu_{6,5}$ & 0.0050 & 0.054 & 0.0405 & - \\
\hline & $\mu_{8,7}$ & 0.0040 & 0.042 & 0.0315 & - \\
\hline \multirow{3}{*}{ Minor human error rates } & $\delta_{2,3}$ & 0.0135 & 0.0120 & 0.012 & - \\
\hline & $\delta_{4,5}$ & 0.0108 & 0.0096 & 0.0096 & - \\
\hline & $\delta_{6,7}$ & 0.0090 & 0.0080 & 0.0080 & - \\
\hline \multirow{4}{*}{ Critical human error rates } & $\gamma_{2,9}$ & 0.00060 & 0.00045 & 0.0075 & - \\
\hline & $\gamma_{4,9}$ & 0.00048 & 0.00036 & 0.0060 & - \\
\hline & $\gamma_{6,9}$ & 0.00040 & 0.00030 & 0.0050 & - \\
\hline & $\gamma_{8,9}$ & 0.00032 & 0.00024 & 0.0040 & - \\
\hline
\end{tabular}

$\mu_{j i}$ : Minimum repairing rate from state $j$ to state $i$ where $i=2 l-1$ and $j=2 l, l=1, \cdots, d$,

$\alpha(t)_{i j}$ : Degradation rate from state $i$ to state $j$ where $i=$ $2 l-1$ to state $j=2 l+1, l=1, \cdots, d$,

$\beta(t)_{i j}$ : PM rate from acceptable state $i$ to previous better operational state $j$ where $i=2 l+1$ and $j \leq 2 l-1, l=1, \cdots, d$ $-1$ $\delta_{i j}$ : Rate of minor human error from state $i$ to state $j$ where $i=2 l$ and $j=2 l+1, l=1, \cdots, d$,

$\gamma_{i j}$ : Rate of critical human error from state $i$ to state $j$ where $i=2 l$ and $j=2 d+m, l=1, \cdots, d$.

The PM activities contain several types of mutually exclusive candidates, which are displayed by dotted lines, and they are different for minor and major maintenance. 
TABLE 3: Transition rates of assemblies with Weibull distribution.

\begin{tabular}{|c|c|c|c|c|c|c|c|}
\hline \multirow{2}{*}{\multicolumn{2}{|c|}{$\begin{array}{l}\text { a: Weibull scale parameter } \\
\text { b: Weibull shape parameter }\end{array}$}} & \multicolumn{2}{|c|}{ ENG } & \multicolumn{2}{|c|}{ UG } & \multicolumn{2}{|c|}{ MTG } \\
\hline & & a & $\mathrm{b}$ & a & $\mathrm{b}$ & a & $\mathrm{b}$ \\
\hline \multirow{4}{*}{ Degradation rates } & $\alpha_{1,3}(t)$ & 600 & 3.2 & 550 & 2.5 & 520 & 3.5 \\
\hline & $\alpha_{3,5}(t)$ & 650 & 3.4 & 590 & 2.7 & 560 & 3.7 \\
\hline & $\alpha_{5,7}(t)$ & 700 & 3.6 & 630 & 2.9 & 600 & 3.9 \\
\hline & $\alpha_{7,9}(t)$ & 800 & 4.0 & 700 & 3.3 & 670 & 4.3 \\
\hline \multirow{6}{*}{ Preventive maintenance rates } & $\beta_{7,1}(t)$ & 400 & 2 & 390 & 1.9 & 350 & 1.9 \\
\hline & $\beta_{7,3}(t)$ & 430 & 1.6 & 410 & 1.7 & 370 & 1.7 \\
\hline & $\beta_{7,5}(t)$ & 450 & 1.2 & 420 & 1.2 & 380 & 1.2 \\
\hline & $\beta_{5,1}(t)$ & 400 & 2 & 390 & 1.9 & 350 & 1.9 \\
\hline & $\beta_{5,3}(t)$ & 430 & 1.6 & 410 & 1.7 & 370 & 1.7 \\
\hline & $\beta_{3,1}(t)$ & 400 & 2 & 390 & 1.9 & 350 & 1.9 \\
\hline
\end{tabular}

TABLE 4: State performance of assemblies.

\begin{tabular}{lcccc}
\hline $\begin{array}{l}\text { Assemblies } \\
\text { States }\end{array}$ & ENG & UG & MTG & OTM \\
\hline 1 & $2,000 \mathrm{hp}$ & $100 \%$ & $100 \%$ & $100 \%$ \\
2 & $0 \mathrm{hp}$ & $0 \%$ & $0 \%$ & $0 \%$ \\
3 & $1,800 \mathrm{hp}$ & $90 \%$ & $80 \%$ & \\
4 & $0 \mathrm{hp}$ & $0 \%$ & $0 \%$ & \\
5 & $1,500 \mathrm{hp}$ & $70 \%$ & $60 \%$ & \\
6 & $0 \mathrm{hp}$ & $0 \%$ & $0 \%$ & \\
7 & $1,000 \mathrm{hp}$ & $30 \%$ & $40 \%$ & \\
8 & $0 \mathrm{hp}$ & $0 \%$ & $0 \%$ & \\
9 & $0 \mathrm{hp}$ & $0 \%$ & $0 \%$ & \\
\hline
\end{tabular}

According to the MSS model depicted in Figure 2, this study establishes a multiobjective MSSPMM with human reliability, wherein mean system unavailability and total maintenance cost are the optimisation objectives. In addition, different combinations of PM activities yield distinct PM candidates and form exploitation spaces for subsequent optimisation processes. An illustrated case related to the helicopter power transmission system that is used to verify the proposed approach is presented below. In this scenario, nonhomogeneous Markov models and related Markov reward models are employed to calculate the MSS performance metrics. The MSS performance calculation involves the following steps.

Step 1. Establish the stochastic models of the helicopter power transmission system.

The system has 13,122 combinatorial states for six assembly state combinations, which deems the solving of nonhomogeneous Markov models unrealistic. For the components, this study utilises the UGF technique to integrate the PMF of each individual assembly to determine the MSS PMF, thereby mathematically representing the dynamic behaviour of the helicopter power transmission system over
TABle 5: Minimum repair costs and PM costs of assemblies.

\begin{tabular}{|c|c|c|c|c|c|}
\hline $\begin{array}{l}\text { Assemblies } \\
\text { Cost }\end{array}$ & & ENG & UG & MTG & OTM \\
\hline \multirow{11}{*}{ Minimum repair cost } & $C_{2,1}$ & 100 & 50 & 80 & 70 \\
\hline & $C_{4,3}$ & 110 & 60 & 90 & - \\
\hline & $C_{6,5}$ & 120 & 75 & 110 & - \\
\hline & $C_{8,7}$ & 130 & 100 & 150 & - \\
\hline & $C_{2,3}$ & 100 & 50 & 80 & - \\
\hline & $C_{4,5}$ & 110 & 60 & 90 & - \\
\hline & $C_{6,7}$ & 120 & 75 & 110 & - \\
\hline & $C_{2,9}$ & 100 & 50 & 80 & - \\
\hline & $C_{4,9}$ & 110 & 60 & 90 & - \\
\hline & $C_{6,9}$ & 120 & 75 & 110 & - \\
\hline & $C_{8,9}$ & 130 & 100 & 150 & - \\
\hline \multirow{6}{*}{ PM cost } & $C_{7,1}$ & 600 & 350 & 500 & - \\
\hline & $C_{7,3}$ & 400 & 200 & 200 & - \\
\hline & $C_{7,5}$ & 200 & 100 & 120 & - \\
\hline & $C_{5,1}$ & 550 & 250 & 400 & - \\
\hline & $C_{5,3}$ & 350 & 120 & 150 & - \\
\hline & $C_{3,1}$ & 500 & 200 & 300 & - \\
\hline
\end{tabular}

time. Thus, the state-transition diagram of each individual assembly is drawn initially, as shown in Figures 3-6. Relatively small-scale nonhomogeneous Markov models in the differential form of the Chapman-Kolmogorov equations corresponding to four different assemblies can be established by compiling the transition intensity matrices that contain the degradation rates, failure rates, minimum repair rates, PM rates, minor human error rates, and critical human error rates for the six assemblies given the PM candidate.

Step 2. Determine the probability mass function for the entire helicopter power transmission system. 
TABLE 6: DOE scheme and experimental results.

\begin{tabular}{|c|c|c|c|c|c|c|c|}
\hline Human reliability levels & $\begin{array}{l}\text { Sets of failure rates and } \\
\text { minimum repair rates }\end{array}$ & $\delta$ & $\gamma$ & $\lambda$ & $\mu$ & $\overline{\mathrm{UA}}$ & Cs \\
\hline \multirow{3}{*}{ I } & 1 & & & 1 & 1 & 0.1321 & 973 \\
\hline & 2 & 0 & 0 & 2 & 0.5 & 0.3161 & 1471 \\
\hline & 3 & & & 0.5 & 2 & 0.0773 & 509 \\
\hline \multirow{3}{*}{ II } & 1 & & & 1 & 1 & 0.2450 & 1087 \\
\hline & 2 & 1 & 0 & 2 & 0.5 & 0.4907 & 1612 \\
\hline & 3 & & & 0.5 & 2 & 0.1349 & 536 \\
\hline \multirow{3}{*}{ III } & 1 & & & 1 & 1 & 0.1586 & 965 \\
\hline & 2 & 0 & 1 & 2 & 0.5 & 0.3482 & 1433 \\
\hline & 3 & & & 0.5 & 2 & 0.0933 & 505 \\
\hline \multirow{3}{*}{ IV } & 1 & & & 1 & 1 & 0.2629 & 1081 \\
\hline & 2 & 1 & 1 & 2 & 0.5 & 0.5054 & 1654 \\
\hline & 3 & & & 0.5 & 2 & 0.148 & 531 \\
\hline
\end{tabular}

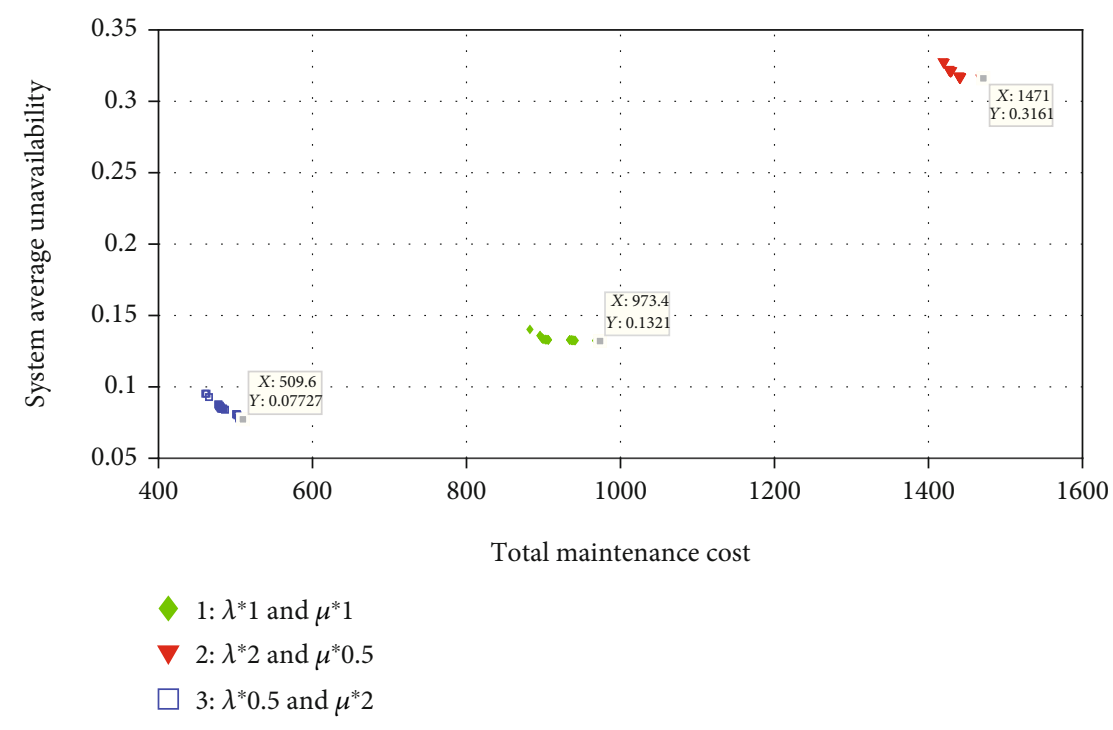

FIGURe 14: Pareto frontier for first-level human reliability.

Solving the nonhomogeneous Markov models for four different assemblies from Step 1 determines their PMFs, which represent the basis of their dynamic behaviour. Then, the PMF of the entire helicopter power transmission system over time is determined using the UGF technique, which involves only simple algebraic calculations; it plays a pivotal role in determining the dynamic behaviour, featuring MSS characteristics.

Step 3. Calculate mean system unavailability and total maintenance cost.

The mean system unavailability for the entire helicopter power transmission system is calculated using the PMF obtained from Step 2, while nonhomogeneous Markov reward models [33] are established for each assembly to determine the total maintenance cost by summation over the individual maintenance cost with a given PM strategy. Next, the mean system unavailability and total maintenance cost are substituted into the established biobjective MSSPMM. Sensitivity analysis is performed to gain further insight regarding the effect of human reliability on the helicopter power transmission system through relevant parameters.

\section{Multiobjective MSSPMM Establishment and Optimisation}

3.1. Multiobjective MSSPMM Establishment. Based on the aforementioned PM model, the established multiobjective MSSPMM can be described as follows.

Objectives:

(1) Minimise mean system unavailability: min UA

(2) Minimise total maintenance cost: min Cs 


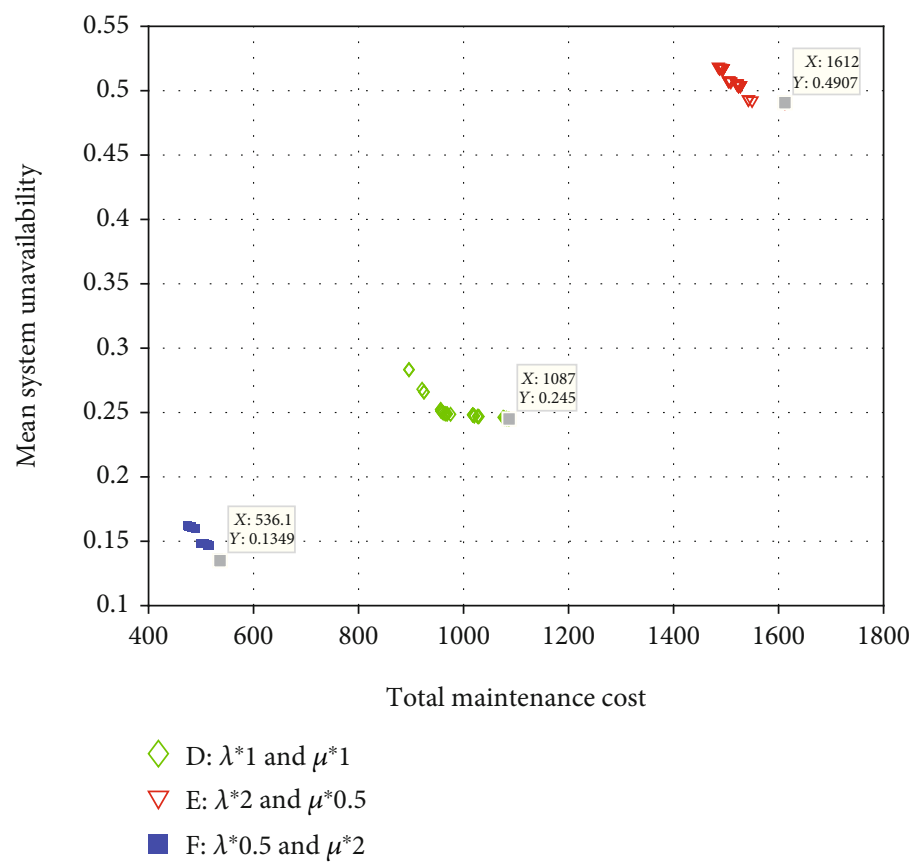

FIgURE 15: Pareto frontier for second-level human reliability.

The functional constraint is given by

$$
\sum_{j=1}^{k_{j}} x_{i, j} \leq 1,
$$

where $\overline{\mathrm{UA}}$, Cs, and $x_{i, j}$ represent the mean system unavailability, total maintenance cost, and binary decision variables for the $i^{\text {th }}$ assembly in the $j^{\text {th }}$ degraded state, totaling $k_{j}$ states, respectively, where a value of one indicates a specific PM action and a value of zero indicates nothing. The constraint in the model is responsible for limiting each degradation state of the assembly to a maximum of one PM action to enable the easy implementation of the PM strategy for lowering the maintenance error with human reliability. Nevertheless, the established PM model can be adapted to meet practical needs through modifications such as altering objective functions or adding appropriate constraints for ensuring that the adapted multiobjective MSSPMM is appropriate.

3.2. Multiobjective MSSPMM with Human Reliability Optimisation. NSGA-III is further adapted to enable the resolution of the established biobjective MSSPMM with human reliability, in which the binary decision variables form a combinatorial optimisation problem. The steps in the customised NSGA-III are presented below.

Step 1. Perform chromosome coding.

A total of 24 distinct binary-code genes produce a distinguished chromosome corresponding to 24 possible PM actions, which constitutes a PM strategy for the helicopter power transmission system. The code is structured based on the No. 1 and No. 2 engines, and the UG and MTG, each of which have six possible PM actions. Thus, there are $2^{24}$ $=16,777,216$ possible PM combinations in the feasible solution space that can be explored to optimise the mean system unavailability and total maintenance cost in parallel.

Step 2. Generate chromosome population.

In this case, the scale of the chromosome population is set at $n=40$. Accordingly, a total of 40 chromosomes coded by different genes are generated through a random mechanism where each chromosome corresponds to a maintenance strategy solution. Then, the chromosome evolution mechanism is executed to determine and approximate the best solution in the entire domain.

Step 3. Implement chromosome-eliminating mechanism for infeasible solutions.

When running NSGA-III, by satisfying the constraint of Equation (1), $\sum_{j=1}^{k_{j}} x_{i, j} \leq 1$, for all chromosomes that correspond to PM solutions; chromosomes that violate the fitness penalty values in terms of cost and unavailability can be excluded from the evolutionary mechanism. The chromosome containing 24 distinct binary-code genes is transformed into a four-gene-subset vector of maintenance activity for implementing the chromosome-eliminating mechanism as outlined below.

(1) Construct a four-gene-subset vector of maintenance activity as $\left[\begin{array}{llllll}32 & 32 & 1 & 32 & 32 & 32\end{array}\right]$, where each element in the gene subset represents the possible maintenance activities in the degraded states of each component 


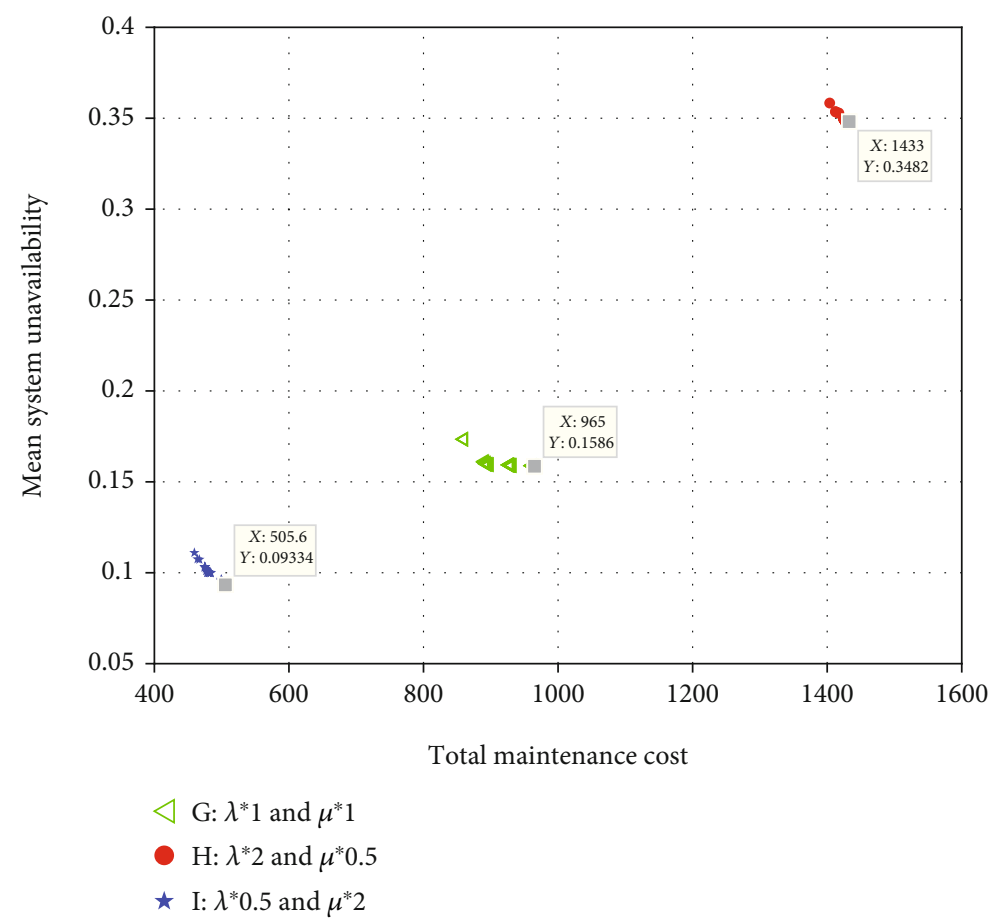

Figure 16: Pareto frontier for third-level human reliability.

(2) Chromosomes that conform to the constraint $\sum_{j=1}^{k_{j}}$ $x_{i, j} \leq 1$ will enter the tailored NSGA-III algorithm evolution mechanism to propagate offspring; in contrast, an extremely poor fitness value will lead to exclusion from the subsequent evolution mechanism

Step 4. Construct reference points.

Reference points are built evenly distributed between the two objectives to facilitate the evolution of the chromosome population toward the Pareto frontier, and a niche preservation technique is used in the GA screening mechanism to maintain the diversity of the chromosome population in each iteration.

Step 5. Propagate offspring chromosome.

Offspring of chromosome $n$ with identical sizes are generated through a random binary crossover along with mutation mechanisms before emerging into the chromosome population $n$, which constitute evolutionary seeds with $2 n$ chromosomes for the subsequent Pareto-dominance screening procedures.

Step 6. Conduct nondominated sorting.

(1) Rank solution sets of chromosomes in order of Pareto dominance. Solutions that are not dominated by any solutions are placed in Rank 1 (the best rank). Solutions that are dominated by Rank
1 solutions only are placed in Rank 2, those dominated by Ranks 1 and 2 only are placed in Rank 3 , etc. Thus, the better the nondominated solution set of chromosomes is, the smaller is the rank number.

(2) According to the rank, the number of transcending chromosomes determined based on the crossover rate is added to the crossover pool for the subsequent genetic evolution. Given that the established PM model mandates that there be at most one PM action in any degraded state, chromosomes violating this constraint are assigned extreme penalty values in terms of the mean system unavailability and total maintenance cost, which ensures that they do not enter the next genetic evolution pool.

\section{Step 7. Generate offspring.}

Response surface methodology is utilised instead of the trial-and-error approach to determine the optimal settings of related parameters, including crossover and mutation rates [34, 35]; this strengthens its solution-searching capabilities. Through the crossover and mutation genetic mechanisms, the offspring are generated randomly with the crossover rate set to 0.8 , mutation rate set to 0.5 , and degree of mutation set to 0.2 . The genetic evolution procedure that includes rank and selection, crossover, and mutation mechanisms is conducted repeatedly, returning to Step 3 until the customised NSGA-III meets the termination condition of 80 generations. 


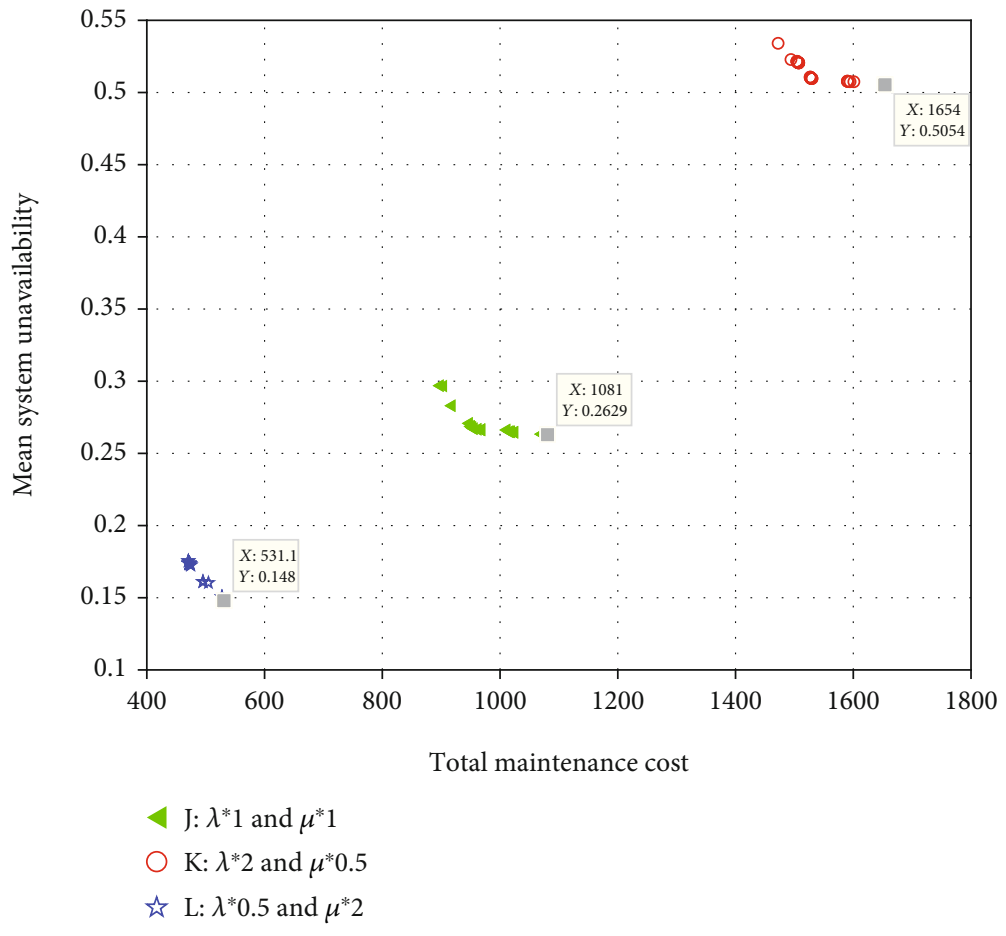

FIgURE 17: Pareto frontier for fourth-level human reliability.

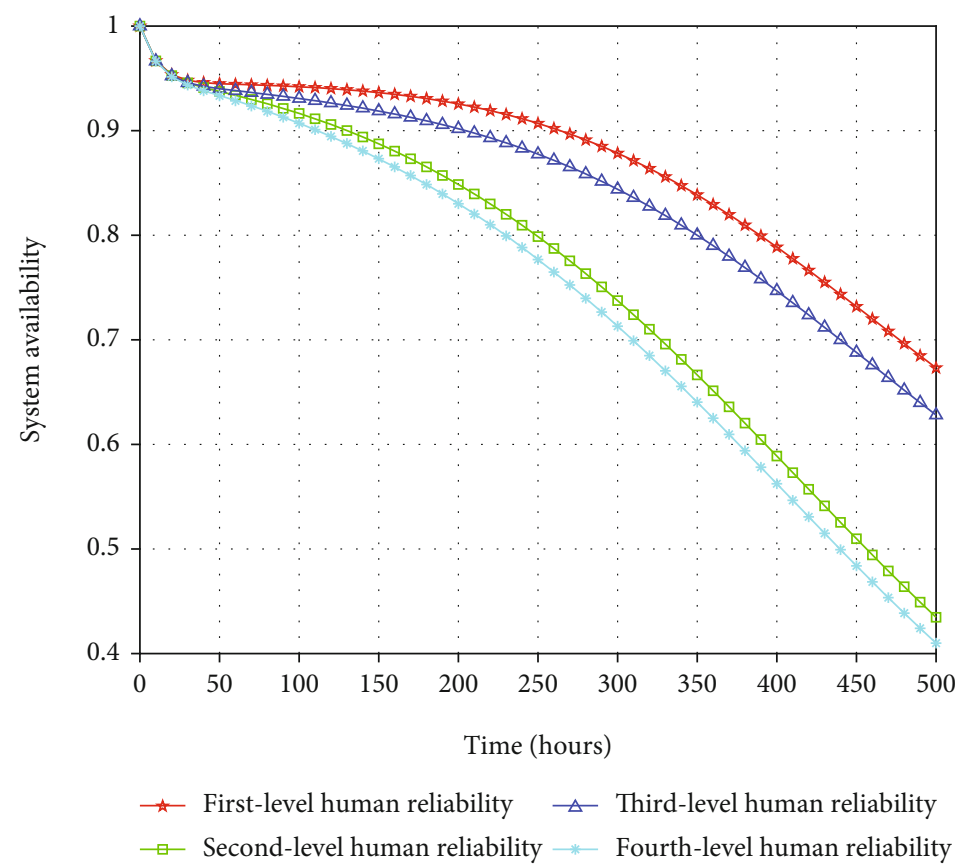

FIGURE 18: System availability trajectory with failure and repair rates unchanged.

Step 8. Output nondominated solutions.

In the illustrated case, the mission time is set to $500 \mathrm{~h}$ and the minimum allowable power for the helicopter power transmission is set to $w=1,800 \mathrm{hp}$. In this case, the Pareto frontier, which comprises the nondominated solution sets in each generation in the spectrum, can be graphed.

\section{Results and Discussion}

Figure 7 shows the diagram of the approaching course, and Figure 8 displays the Pareto frontier with 17 optimal nondominated solutions. Figure 8 indicates that, among the 17 solutions, the minimum mean system unavailability is 0.2668 with a total maintenance cost of 1008 , whereas the 


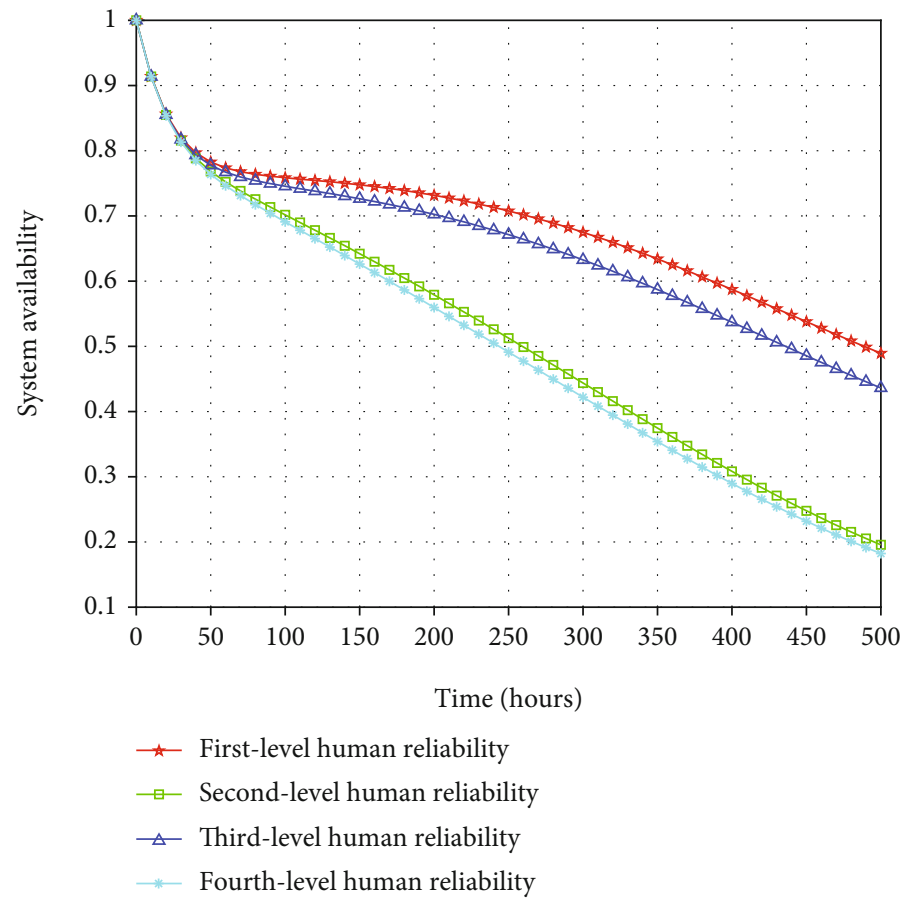

Figure 19: System availability trajectory with deteriorated failure and repair rates.

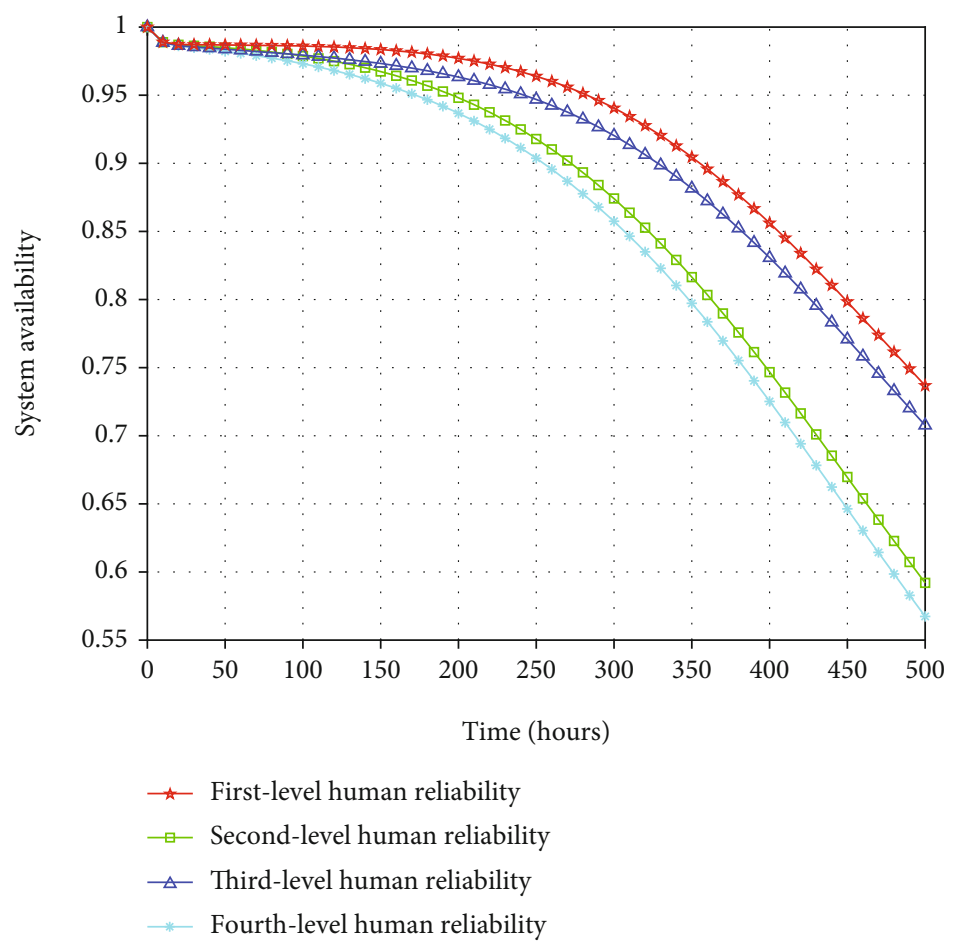

FIGURE 20: System availability trajectory with improved failure and repair rates.

minimum total maintenance cost is 884 with a mean system unavailability of 0.3013 . For a helicopter power transmission system requiring a mean system unavailability below 0.28 , three distinct PM alternatives meet the requirements, as summarised in Table 1. Figure 9 displays the system availability trajectory with the time for the three PM alternatives.
Further, restricting the total maintenance cost to below 1000, the first two PM alternatives are candidates with the first having a lower total maintenance cost (950) compared to the second. Figures 10-13 show the state-transition diagrams of the No. 1 and No. 2 engines and those of the UG and MTG related to the first PM alternative. 
Table 2 lists the failure rates, minimum repair rates, minor human error rates, and critical human error rates, all exponentially distributed. Table 3 presents the degradation and $\mathrm{PM}$ rates with a Weibull distribution for four different assemblies of the power transmission system. Table 4 summarises the state performance of each assembly, and Table 5 presents the minimum repair costs and PM costs.

4.1. Sensitivity Analysis. This study employed a sensitivity analysis with a design of experiment (DOE) scheme to further explore the extent to which human reliability impacts the system availability and total maintenance cost, as shown in Table 6 , where $\delta$ represents the intensity of descending into the next worst state and $\gamma$ indicates entering a complete failure state. The DOE scheme includes the combined fourlevel human reliability under three distinct sets of failure rates $(\lambda)$ and minimum repair rates $(\mu)$ that remain unchanged, deteriorate, or improve in terms of failure and repair with unchanged degradation $(\alpha(t))$ and $\mathrm{PM}$ rates.

The first-level human reliability simulates a lack of human error occurrence, the second-level reliability simulates minor human error with an occurrence rate of $\delta$, the third-level reliability simulates critical human error with an occurrence rate of $\gamma$, and the fourth-level reliability accounts for the impacts of both $\delta$ and $\gamma$. Accordingly, a total of 12 experiments were conducted, and the results in terms of the mean system unavailability and total maintenance cost are presented in Table 6. Figures 14-17 show the Pareto frontiers in terms of the mean system unavailability and total maintenance cost for four-level human reliability under three distinct combinations of failure rate and minimum repair rate. Further, Figures 18-20 present the system availability trajectory for three distinct combinations of the failure rate and minimum repair rate under a four-level human reliability with time.

Figures 14-17 indicate that the optimal solution sets on the Pareto frontiers with improved failure and minimum repair rate combinations outperforming deteriorated failure and repair rate combinations; results with unchanged failure and minimum repair rates are between those mentioned above. This structural pattern is consistent with our mathematical expectations.

Further, system availability in the span of the mission time decreases in synchronisation with human reliability. The fourth level with the strongest intensity of human error presents the lowest availability trajectory, regardless of which combination of failure rates and minimum repair rates is used, as indicated by Figures 18-20. The first level with no human error outperforms the other three different extents of human error; this structural tendency demonstrates the importance of addressing human reliability in the engineering industry, where this factor plays an essential role.

\section{Conclusions}

The conclusions of the study are summarised as follows.

(1) The proposed MSSPMM is particularly appropriate for repair works that involve highly skilled personnel whose human error can lead to critical negative con- sequences such as the deterioration of system performance or even complete system failure that inflicts harm to personnel

(2) The sensitivity analysis provides further insight into the extent to which human reliability affects the entire system availability and total maintenance cost, which can subsequently be utilised to improve personnel arrangements to fulfil task requirements such as ensuring system availability, reduction of damage or loss because of failure, and reduction of maintenance costs

(3) An improved NSGA-III algorithm was developed to solve the established constrained multiobjective MSSPMM, in which the chromosome-screening mechanism was initiated in accordance with the constraint beyond the combinatorial GA coding. The MSSPMM was constrained to allow at most one maintenance activity in degradation states from the perspective of components

(4) By modelling the mean system unavailability and total maintenance cost as a biobjective problem, the proposed approach can be easily and practically adapted to alternative scenarios simply by adding other objectives and/or constraints that are aligned to the practical requirements

(5) Although the study focuses on the MSS reliability model, which is becoming increasingly popular in modern-day instrumentation because of the advanced technology employed, as demonstrated with a helicopter power transmission system normally equipped with monitoring devices, the proposed approach can be expanded to the binary reliability model considering human reliability. Mathematically, solving an MSS reliability model is more complicated than solving its binary counterparts; therefore, this study tailored the NSGA-III algorithm to optimise the proposed multiobjective MSSPMM with human reliability

Future studies can examine models wherein human reliability affects the overall system performance, particularly in cases where the system failure might cause severe problems or even death, such as in the aviation sector and power plants.

\section{Data Availability}

The data used to support the findings of this study are included within the article.

\section{Conflicts of Interest}

The authors declare no conflicts of interest.

\section{Acknowledgments}

This research was supported by the Ministry of Science and Technology of the Republic of China (MOST 107-2221-E606-008). 


\section{References}

[1] Y. Guo, Y. Sun, X. Yang, and Z. Wang, "Flight safety assessment based on a modified human reliability quantification method," International Journal of Aerospace Engineering, vol. 2019, Article ID 2812173, 12 pages, 2019.

[2] G. Heo and J. Park, "A framework for evaluating the effects of maintenance-related human errors in nuclear power plants," Reliability Engineering and System Safety, vol. 95, no. 7, pp. 797-805, 2010.

[3] S. Kalaiarasi, A. Merceline Anita, and R. Geethanjalii, "Analysis of system reliability using Markov technique," Global Journal of Pure and Applied Mathematics, vol. 13, no. 9, pp. 52655273, 2017.

[4] E. Zaitseva, V. Levashenko, J. Rabcan, and E. Krsak, "Application of the structure function in the evaluation of the human factor in healthcare," Symmetry, vol. 12, no. 1, p. 93, 2020.

[5] E. Hollnagel, Cognitive Reliability and Error Analysis Method (CREAM), Elsevier, 1998.

[6] S. M. Asadzadeh and A. Azadeh, "An integrated systemic model for optimization of condition-based maintenance with human error," Reliability Engineering and System Safety, vol. 124, pp. 117-131, 2014.

[7] M. Masson and Y. Koning, "How to manage human error in aviation maintenance? The example of a JAR 66-HF education and training programme," Technology \& Work, vol. 3, no. 4, pp. 189-204, 2001.

[8] J. C. Chen and V. F. Yu, "Relationship between human error intervention strategies and unsafe acts: the role of strategy implementability," Journal of Air Transport Management, vol. 69, pp. 112-122, 2018.

[9] N. C. Caballé, I. T. Castro, C. J. Pérez, and J. M. Lanza-Gutiérrez, "A condition-based maintenance of a dependent degradation-threshold-shock model in a system with multiple degradation processes," Reliability Engineering and System Safety, vol. 134, pp. 98-109, 2015.

[10] I. T. Castro, N. C. Caballé, and C. J. Pérez, “A condition-based maintenance for a system subject to multiple degradation processes and external shocks," International Journal of Systems Science, vol. 46, no. 9, pp. 1692-1704, 2015.

[11] Q. Zhai, Z. S. Ye, J. Yang, and Y. Zhao, "Measurement errors in degradation-based burn-in," Reliability Engineering \& System Safety, vol. 150, pp. 126-135, 2016.

[12] M. Manoharan and V. G. Nair, "Evaluation of system performance measures of multi state degraded system with minimal repair," Reliability: Theory \& Applications, vol. 12, no. 1, pp. 76-83, 2017.

[13] S. Alaswad and Y. Xiang, "A review on condition-based maintenance optimization models for stochastically deteriorating system," Reliability Engineering and System Safety, vol. 157, pp. 54-63, 2017.

[14] L. Jafari and V. Makis, "Joint optimization of lot-sizing and maintenance policy for a partially observable two-unit system," International Journal of Advanced Manufacturing Technology, vol. 87, no. 5-8, pp. 1621-1639, 2016.

[15] J. Son, S. Zhou, C. Sankavaram, X. Du, and Y. Zhang, "Remaining useful life prediction based on noisy condition monitoring signals using constrained Kalman filter," Reliability Engineering and System Safety, vol. 152, pp. 38-50, 2016.

[16] Z. Zhao, B. Liang, X. Wang, and W. Lu, "Remaining useful life prediction of aircraft engine based on degradation pattern learning," Reliability Engineering and System Safety, vol. 164, pp. 74-83, 2017.

[17] K. S. Moghaddam, "Preventive maintenance and replacement optimization on CNC machine using multiobjective evolutionary algorithms," International Journal of Advanced Manufacturing Technology, vol. 76, no. 9-12, pp. 2131-2146, 2015.

[18] A. Salmasnia, E. Ameri, A. Ghorbanian, and H. Mokhtari, "A multi-objective multi-state degraded system to optimize maintenance/repair costs and system availability," Scientia Iranica, vol. 24, no. 1, pp. 355-363, 2017.

[19] V. P. Koutras, S. Malefaki, and A. N. Platis, "Optimization of the dependability and performance measures of a generic model for multi-state deteriorating systems under maintenance," Reliability Engineering and System Safety, vol. 166, pp. 73-86, 2017.

[20] B. Ombuki and B. J. Ross, "Multi-objective genetic algorithms for vehicle routing problem with time windows," Applied Intelligence, vol. 24, no. 1, pp. 17-30, 2006.

[21] R. H. Bhesdadiya, I. N. Trivedi, P. Jangir, N. Jangir, and A. Kumar, "An NSGA-III algorithm for solving multiobjective economic/environmental dispatch problem," Cogent Engineering, vol. 3, no. 1, 2016.

[22] C. Guo, C. Ye, Y. Ding, Z. Lin, and P. Wang, "Risk-based many-objective configuration of power system fault current limiters utilising NSGA-III," IET Generation, Transmission \& Distribution, vol. 14, no. 23, pp. 5646-5654, 2020.

[23] X. Yang and Y. Zhang, "Optimal transmission line assignment considering reliabilities and assignment cost in the multi-state network," IEEE Access, vol. 8, pp. 105690-105700, 2020.

[24] Y. Li, Y. Kou, and Z. Li, "An improved nondominated sorting genetic algorithm III method for solving multiobjective weapon-target assignment part I: the value of fighter combat," International Journal of Aerospace Engineering, vol. 2018, Article ID 8302324, 23 pages, 2018.

[25] X. Ni, Z. Dong, W. Xie, W. Jia, C. Duan, and H. Yao, "Research on the multi-objective cooperative competition mechanism of Jinsha river downstream cascade reservoirs during the flood season based on optimized NSGA-III," Water, vol. 11, no. 4, p. 849, 2019.

[26] X. Bi and C. Wang, "An improved NSGA-III algorithm based on objective space decomposition for many-objective optimization," Soft Computing, vol. 21, no. 15, pp. 4269-4296, 2017.

[27] Q. Liu, X. Liu, J. Wu, and Y. Li, “An improved NSGA-III algorithm using genetic K-means clustering algorithm," IEEE Access, vol. 7, pp. 185239-185249, 2019.

[28] J. H. Zheng, C. Q. Wu, J. Huang, Y. Liu, and Q. H. Wu, "Multiobjective optimization for coordinated day-ahead scheduling problem of integrated electricity-natural gas system with microgrid," IEEE Access, vol. 8, pp. 86788-86796, 2020.

[29] Q. Chao, "Multi-objective evolutionary algorithms are still good: maximizing monotone approximately submodular minus modular functions," Evolutionary Computing, pp. 128, 2020.

[30] A. A. Zahra, M. M. Paydar, S. H. Nasseri, and I. Mahdavi, “A meta-heuristic approach supported by NSGA-II for the design and plan of supply chain networks considering new product development," Journal of Industrial Engineering International, vol. 14, pp. 95-109, 2018.

[31] R. Behmanesh, I. Rahimi, and A. H. Gandomi, "Evolutionary many-objective algorithms for combinatorial optimization 
problems: a comparative study," Archives of Computational Methods in Engineering, vol. 28, no. 2, pp. 673-688, 2021.

[32] A. Lisnianski, I. Frenkel, and L. Khvatskin, "On Birnbaum importance assessment for aging multi-state system under minimal repair by using the $\mathrm{L}_{\mathrm{z}}$-transform method," Reliability Engineering and System Safety, vol. 142, pp. 258-266, 2015.

[33] A. Lisnianski, I. Frenkel, and Y. Ding, Multi-State System Reliability Analysis and Optimization for Engineers and Industrial Managers, Springer, 2010.

[34] C. H. Wang and S. W. Tsai, "Optimizing bi-objective imperfect preventive maintenance model for series-parallel system using established hybrid genetic algorithm," Journal of Intelligent Manufacturing, vol. 25, no. 3, pp. 603-616, 2014.

[35] D. C. Montgomery, Design and Analysis of Experiments, Wiley, U.S.A, NY, 6th edition, 2005. 